\title{
A virtual reality approach to the Trier Social Stress Test: Contrasting two distinct protocols
}

\author{
Eva Montero-López • Ana Santos-Ruiz • \\ M. Carmen García-Ríos • Raúl Rodríguez-Blázquez • \\ Miguel Pérez-García • María Isabel Peralta-Ramírez
}

Published online: 12 February 2015

(C) Psychonomic Society, Inc. 2015

\begin{abstract}
Virtual reality adaptations of the Trier Social Stress Test (TSST-VR) constitute useful tools for studying the physiologic axes involved in the stress response. Here, we aimed to determine the most appropriate experimental approach to the TSST-VR when investigating the modulation of the axes involved in the stress response. We compared the use of goggles versus a screen projection in the TSST-VR paradigm. Fortyfive healthy participants were divided into two groups: the first one (goggles condition; 13 females, 11 males) wore goggles while performing the TSST-VR; the second (screen condition; 15 females, six males) was exposed to the TSST-VR projected on a screen. Sympathetic reactivity to stress was measured by continuously recording skin conductance (SC), while the hypothalamic-pituitary-adrenal axis (HPA) was evaluated by sampling salivary cortisol throughout the experiment. At the end of the task, there was an increase in SC and cortisol level for both means of delivering the TSST-VR, although the increase in $\mathrm{SC}$ was greater in the goggles
\end{abstract}

E. Montero-López $(\triangle) \cdot$ M. Pérez-García $\cdot$ M. I. Peralta-Ramírez Department of Clinical Psychology, Faculty of Psychology, University of Granada, Campus de Cartuja s/n, 18071 Granada, Spain

e-mail: evamonterolopez1983@gmail.com

A. Santos-Ruiz

Department of Health Psychology, Faculty of Health Sciences,

University of Alicante, Alicante, Spain

M. C. García-Ríos

Department of Physical Therapy, Faculty of Health Sciences,

University of Granada, Granada, Spain

R. Rodríguez-Blázquez

Health Center of Villarrodrigo, Jaén, Spain

M. Pérez-García • M. I. Peralta-Ramírez

Mind, Brain and Behavior Research Center (CIMCYC),

Granada, Spain condition, while salivary cortisol was comparable in both groups. Immersion levels were reportedly higher in the screen presentation than in the goggles group. In terms of sex differences, females experienced greater involvement and spatial presence, though comparatively less experienced realism, than their male counterparts. These findings help us determine which protocol of the TSST-VR is most suitable for the stress response under study. They also emphasize the need to consider the sex of participants, as males and females show distinct responses in each protocol.

Keywords TSST · Virtual reality ·

Hypothalamic-pituitary-adrenal axis $\cdot$ Sympathetic activation $\cdot$ Salivary cortisol

\section{Introduction}

The Trier Social Stress Test (TSST) is an experimental paradigm used as a tool in investigating the psychobiologic stress response in the laboratory, as it activates the hypothalamicpituitary-adrenal axis (HPA) (Kirschbaum, Pirke, \& Hellhammer, 1993). The response is reflected in an increase in heart rate, blood pressure, and adrenaline and cortisol levels, as well as in a negative effect on mood (Al'Absi et al., 1997; Nicolson, Storms, Ponds, \& Sulon, 1997). In the traditional TSST, as proposed by Kirschbaum et al. (1993), participants have to prepare a speech, claiming they are suitable for a given job appointment, articulate the speech in front of an audience, and subsequently complete an arithmetic task in public. The audience is generally composed of people who had been previously trained in neutral verbal and non-verbal behaviors (Foley \& Kirschbaum, 2010). 
Vinkers et al. (2013) recorded psychophysiologic variables to investigate the effects of stress on core and peripheral body temperature in humans. They used the TSST and obtained distinct response patterns in men (i.e., increased cheek temperature) and women (i.e., decreased nasal skin temperature).

The original TSST has been utilized, since its inception, to measure stress reactivity in diverse populations such as children (TSST-C; Buske-Kirschbaum et al., 1997), middle-aged adults (Fiocco, Joober, \& Lupien, 2007), and retired people (Kudielka et al., 1998), as well as in various pathologies, such as psychiatric patients (Brenner et al., 2009), metabolic syndrome (Chrousos, 2000), systemic hypertension (Esler et al., 2008), systemic lupus erythematosus (Pawlak et al., 1999; Santos-Ruiz et al., 2010), and myalgias (Sjörs et al., 2010). Likewise, the TSST has been used to test the relationship between stress and a variety of psychologic variables, such as depression (Parker, Schatzberg, \& Lyons, 2003), social anxiety (Shirotsuki et al., 2009), and personality traits (Kirschbaum, Bartussek, \& Strasburger, 1992; Pruessner et al., 1997).

The adaptation of the TSST into a virtual reality (VR) environment elicits a significant physiologic response, thus solving some of the common TSST limitations that come from constraints both in the design of different environments or set-ups and from the assembling of acceptably large audiences for each experimental session, given that the magnitude of the stress response depends in part on the size of the audience. An additional limitation results from the cost of TSST implementation, as it is necessary to have separate rooms for experimental subjects and audiences, a certain time is spent in training audience participants to show neutral behaviors in response to subjects' performance, and audiences may be unreliable and show distinct behaviors between one participant and another. Therefore, it would seem that the TSST in the virtual environment constitutes a viable alternative to the usual forms of presentation of stress tasks (Kotlyar et al., 2008).

Virtual reality tasks are increasingly used in the laboratory setting, given their convenience and efficacy in providing three-dimensional (3D) computerized environments and setups with which to study various pathologies and their associated responses (e.g., stress). In reviewing recent research on the application of VR to the treatment of mental disorders, Malbos, Boyer, and Lançon (2013) concluded that there was sufficient evidence to attest to the efficacy of this methodology in the assessment and treatment of distinct mental disorders.

In a related investigation, Kelly, Matheson, Martinez, Merali, and Anisma (2007) utilized the TSST-VR, as delivered by Virtually Better Inc., Atlanta, GA, in which a previously recorded virtual audience was presented in 3D. Results indicated that giving a speech and carrying out an arithmetic task in front of a virtual audience produced a significant increase in salivary cortisol (30 \%), even though the increase was greater when both tasks were conducted in front of a real audience $(90 \%)$.

In their study, Kotlyar et al. (2008) demonstrated that the TSST-VR resulted in a physiologic response with significant increases observed in response to each stress task in systolic and diastolic blood pressure and heart rate, a finding that suggests the TSST-VR constitutes a viable alternative to previous TSST methodologies, with the additional advantages of reducing variability in the mode of presentation, and decreasing human and financial costs.

Several studies have used the TSST-VR in recent years. For instance, Jönsson et al. (2010) used a CAVETM system developed at the Electronic Visualization Laboratory of the University of Illinois in Chicago, which facilitates total immersion by means of a VR room, a head-tracking system, and passive stereoscopy. This study's main results center on the habituation of the various measures of sympathetic activity obtained in response to the second implementation of the TSST-VR. On the first performance of the TSST-VR there was an $88 \%$ increase of salivary cortisol with respect to baseline levels, which was habituated in the second session.

Another version of the TSST-VR was implemented by Santos Ruiz et al. (2010) by projecting a 3D virtual audience onto a screen. Results showed that this version of the TSSTVR resulted in significant sympathetic activation, as well as activation of the HPA axis in the majority of participants. It was, therefore, concluded that this form of the TSST-VR stimulated the main axes involved in the stress response.

Given the results obtained to date, it seems that the TSSTVR constitutes a useful technique in the study of the activation of the basic axes involved in the stress response. Considering the advantages of this way of presenting the TSST paradigm, it would be beneficial to further investigate which methodologic variations produce greater or lesser immersion in the virtual environment, as we hypothesize that the level of immersion will determine the level of activation of the physiologic axes involved in the stress response. Additionally, it would be worthwhile to explore whether there are sex differences in the stress response brought about by the TSST-VR, as traditional TSST studies have shown that this task results in larger increases of salivary cortisol in men than in women (Foley \& Kirschbaum, 2010; Kirschbaum et al., 1993; Williams, Hagerly, \& Brooks, 2004).

Annerstedt et al. (2013) carried out a pilot study in which a VR environment was used with two distinct objectives; first, producing physiologic stress by means of the TSST-VR, and, secondly, comparing physiologic recovery in different virtual environments after inducing the stress response. Their results showed that the TSST-VR activates the HPA axis and that recovery from the induced stress response varied as a function of the type of virtual environment to which participants were exposed. 
Previous studies of the TSST in the virtual environment have differed in the way they projected 3D images, with some projecting images onto a screen and others using $3 \mathrm{D}$ goggles.

Morina, Brinkman, Hartanto, and Emmelkamp (2014) examined levels of anxiety and sense of presence during exposure to two types of virtual environments: a head-mounted display (HMD) with motion tracker and stereoscopic view, and a one-screen projection-based VR display. Their results suggest that both VR displays were equally effective in evoking moderate levels of anxiety, although the HDM display appeared to produce a stronger sense of presence.

We have explored the potential differences between studies of the TSST-VR paradigm that have used goggles and those that have used a projection screen, and it appears that none of them show a conclusive advantage of using one method over the other in terms of activating the axes of the stress response. Thus, our objectives were twofold. First, we aimed to investigate which of two TSST-VR protocols induced greater sympathetic activation, as measured by skin conductance, and HPA axis activation, as measured by secretion of salivary cortisol. Both protocols included a 3D virtual audience but, while one was presented on a large screen, the other was presented by virtual goggles. A second objective in this study was to examine whether there were sex differences in the activation of either axis, as is the case in the traditional TSST, and whether they differed according to the two TSSTVR protocols.

\section{Method}

\section{Participants}

Forty-five students attending the University of Granada volunteered their participation in this study. They were divided into two groups, according to which protocol of the TSSTVR was used: $3 \mathrm{D}$ goggles or a large screen projection. The goggles group included 13 females and 11 males, with a mean age of 21.9 years $(\mathrm{SD}=5)$ and 14 years of schooling on average $(\mathrm{SD}=0)$. The screen group contained 15 females and six males, with a mean age of 20.4 years $(\mathrm{SD}=1.25)$ and 14 years of schooling on average $(\mathrm{SD}=0)$.

The following were the exclusion criteria, adopted because of their potentially negative effect on cortisol levels (Williams et al., 2004): hypertension, heart disease, obesity, clinical diagnosis of depression or anxiety, personality disorders, and substance use (i.e., amphetamines, methadone, barbiturates, or muscle relaxants). We also recorded sleep duration and excluded any participant that had not slept a minimum of 4 hours on the night prior to the experiment. Participants provided data on sleep duration as part of the semi-structured interview.
On arrival at the laboratory, participants were given information about the experiment and asked to sign an informed consent form approved by the Ethics Committee of the University of Granada and performed according to the recommendations of the Declaration of Helsinki. Immediately afterwards, they were administered the semi-structured interview.

\section{Instruments}

The questionnaires below provided meaningful covariates that could be used in statistical analyses of the psychophysiologic measures and cortisol.

Semi-structured interview Including sociodemographic data, daily life and sleep habits, medication, menstrual cycle, and history of psychiatric or psychologic treatment.

Stress Vulnerability Inventory (SVI) (Beech, Burns, \& Scheffield, 1986; Spanish adaptation, validated by RoblesOrtega, Peralta-Ramirez, \& Navarrete-Navarrete, 2006). This instrument consists of 22 items and evaluates the individual's predisposition to be affected by perceived stress. The Spanish adaptation by Robles-Ortega et al. (2006) shows a Cronbach's alpha of 0.87 . As for convergent validity, the results show a significant positive correlation $(\mathrm{p}<0.01)$ with the following assessment scales: STAI-R, Beck Depression Inventory, Somatic Symptom Scale, and Survey of Recent Life Experiences (SRLE).

Perceived Stress Scale (PSS) (Cohen, Kamarak, \& Mermeistein, 1983; Spanish adaptation by Remor \& Carrobles, 2001) The PSS is a self-report scale used to evaluate perceived stress level and the degree to which people find their lives unpredictable, uncontrollable, or overwhelming (aspects that contribute to stress). It consists of 14 items with five response alternatives. The highest score corresponds to the highest perceived stress level. The Spanish version of the PSS (14 items) has adequate reliability (internal consistency $=$ 0.81 and test-retest $=0.73$ ), concurrent validity, and sensitivity (Remor, 2006). Here, we have considered those scores over 22 (i.e., the mean score for the Spanish population; Remor \& Carrobles, 2001) as reflecting high levels of perceived stress.

SCL-90-R Symptoms Inventory (Derogatis, 1994; Spanish adaptation by Gonzalez de Rivera \& De las Cuevas, 1988) We used this instrument to rule out potential psychopathology in the participants. This self-report questionnaire was developed to assess symptoms of psychopathology and includes 90 items with five response alternatives (0-4) on a Likert scale. Subjects respond according to how they have felt within the past seven days, including the day the inventory is administered. The inventory is scored and interpreted according to nine main dimensions (somatization, obsessive-compulsive 
symptoms, interpersonal sensitivity, depression, anxiety, hostility, phobic anxiety, paranoid ideation, and psychoticism) and three global indices of psychologic distress (Global Severity Index [GSI], Positive Symptom Total [PS], and Positive Symptom Distress Index [PSDI]). In this study, we analyzed these last three global indices. This instrument is thought to have satisfactory reliability and validity (De las Cuevas et al., 1991).

Igroup Presence Questionnaire (IPQ; Schubert, Friedmann, $\&$ Regenbrecht, 2001) The IPQ is used to measure the sense of presence experienced in a virtual environment. The questionnaire was used to rule out the possibility that potential differences between groups could be attributable to variations in the sense of presence experienced by participants within the virtual environment. IPQ responses range from -3 (=totally disagree) to +3 (=totally agree). The IPQ includes a total of 14 items, consisting of a global scale (with scores between -3 and 3) and three subscales: spatial presence (i.e., the sense of being physically present in the virtual environment), with scores between -15 and 15 ; involvement (i.e., the attention devoted to the virtual environment and the degree of involvement experienced), with scores between -12 and 12 ; and $e x-$ perienced realism (i.e., the subjective experience of realism in the virtual environment), with scores between -12 and 12 . Reliability data are not yet available for the Spanish population.

Virtual reality version of the Trier Social Stress Test (TSST$V R$ ) This is a virtual-reality adaptation of the traditional TSST. It includes a computer program that enables the design of a 3D audience, which can be projected into a set of VR goggles (eMagin Z800 3DVisor) with a viewing equivalent of a 105-in diagonal movie screen viewed at $12 \mathrm{ft}$, with a $40^{\circ}$ field of view and stereoscopic image with a $800 \times 600$ triad pixels per display (http://www.3dvisor.com/) connected to a computer or, alternatively, can be projected onto a screen.

Pertaub, Slater, and Barker (2002) showed that participants that are exposed to three virtual audiences whose behavior is different toward the speaker (i.e., neutral, positive, or negative) generate distinct responses in the speaker. In the present study, all participants were exposed to the same type of audience, in identical VR and protocol conditions. Presentations were all stereoscopic, since Ling, Brinkman, Nefs, Qu, and Heynderickx (2012) had shown that stereoscopic VR presentations improved the sense of spatial presence in the case of public speaking. Sound volume and audience behavior were the same for all presentations.

World Viz (http://www.worldviz.com/industries/academic/ neuroscience\#Starter_Kit) was adopted as the software of choice, as it provides VR environments that can be integrated with fMRI signals, biologic responses, motiontracking input, and 3D rendering. The Vizard program, with its high-level scripting language, affords the creation of interactive $3 \mathrm{D}$ content and the integration of biologic signals.

The screen was $2.21 \mathrm{~m}$ wide and $2.30 \mathrm{~m}$ high, with a $44^{\circ}$ field of view and stereoscopic image of $1600 \times 1200$ triad pixels per display and a distance of $1.75 \mathrm{~m}$ from the participant.

Both VR delivery protocols require that participants wear earphones to listen to the sounds coming from the virtual environment, as well as a microphone to mislead them into thinking that their speech will be recorded and thus enhance the threatening effect of the social evaluation component and the participants' level of psychosocial stress. Once the participants were ready to start the experiment, they were informed that the task included four different phases. During the 3-min initial phase, participants were instructed to relax and remain still. This phase was designated as the period of psychophysiologic adaptation to the virtual environment (baseline of skin conductance). Under both experimental conditions, participants were seated. In the Screen Projection condition, they faced a screen showing a 3D image of a stage curtain, while in the Goggles condition, the same image was projected by means of virtual goggles. The second phase, labeled the anticipatory stress period, lasted $5 \mathrm{~min}$, during which participants were required to prepare a speech, describing their positive and negative traits, to be delivered in front of the virtual audience. The third phase, also of $5 \mathrm{~min}$ in duration, was called the speech delivery period. During this period, the curtain came up, the virtual audience appeared (Fig. 1), and participants had to begin delivering their speeches. They were instructed to speak for the entire $5 \mathrm{~min}$ without interruption and attend to the form and content of the speech, as they would greatly determine the audience's response to their speech. The end of the speech constituted the beginning of the last phase of the stressful task, the arithmetic task, based on the Paced Auditory Serial Addition Test (PASAT) in which participants were asked to add two consecutive numbers (i.e., the number they just heard and the one that just preceded it), delivered by means of an auditory recording.

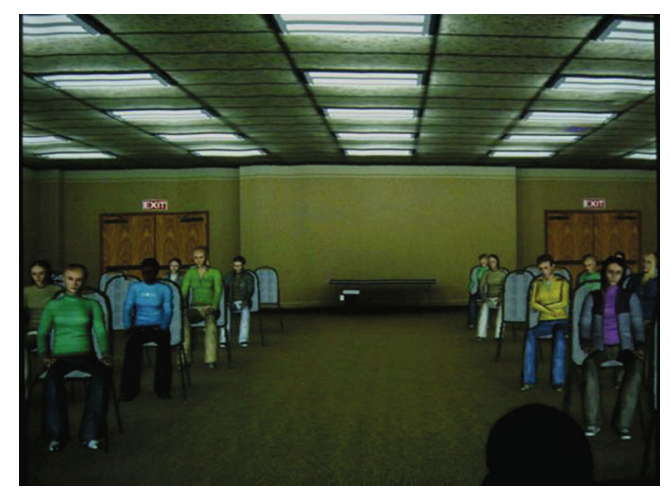

Fig. 1 Three-dimensional virtual audience as projected onto a large screen 


\section{Procedure}

The study was scheduled according to the diurnal cortisol curve, between 3:00 pm and 6:00 pm, when levels of salivary cortisol are more stable. Although cortisol levels are reportedly stable between 2:00 pm and 4:00 pm (Kudielka \& Kirschbaum, 2005), Spanish metabolic and circadian rhythms could differ from those of other European countries, due to the greater number of light hours and the distinctive schedule of meals. Therefore, following a pilot study, Santos-Ruiz et al. (2010) determined in a Spanish sample that cortisol levels were stable between 3:00 pm and 6:00 pm, so all participants were tested in the afternoon, within this temporal window.

Once the participants arrived at the laboratory, they were given information about the experiment and a consent form to be read and signed. Afterwards, they were interviewed and completed the following questionnaires: SV, PSS, and SCL-90-R.

Next, participants were fitted with electro-dermal electrodes to record their skin conductance responses at various time intervals (see assessment of the sympathetic reactivity section below).

Immediately afterwards, the TSST-VR was explained to the participant, the first salivary cortisol sample (pre-exposure cortisol) was collected and the stressful task was started (anticipatory stress, a situation of speech delivery and arithmetic task). Right after the end of arithmetic task, the second cortisol sample (post-exposure cortisol) was collected and the degree of immersion into the VR environment was assessed by administering the IPQ. Ten and $20 \mathrm{~min}$ later, the third (postexposure cortisol +10') and fourth (post-exposure cortisol + 20') cortisol samples, respectively, were taken. Participants were given interior design magazines to leaf through in the interval between the third and fourth drawing of salivary cortisol. A diagram of the protocol of the TSST-VR is shown in Fig. 2.

At the end of the experiment, participants are told that their speech has not been recorded and that the objective of the procedure was to elicit a stress response and not to have their performance evaluated in the speech and arithmetic tasks.

Assessment of the physiologic axes involved in the stress response

Assessment of the sympathetic reactivity Skin conductance was recorded by means of two standard-size $\mathrm{Ag} / \mathrm{AgCl}$ electrodes, with isotonic electrolyte gel placed on the palm of the nondominant hand. Subsequently, data were recorded during visualization of the virtual scenarios, and consisted of a 3-min baseline period prior to the TSST-VR (period of psychophysiologic adaptation to the virtual environment) and continued recording throughout the anticipatory stress $(5 \mathrm{~min})$ and speech delivery ( $5 \mathrm{~min}$ ) periods. Recording of skin conductance ended right after the conclusion of the speech delivery task (see Fig. 2). Since one of the aims of the study was to test the sympathetic reactivity evoked during the speech delivery task under the two experimental conditions, we chose to record measures of skin conductance because they provided a measure of sympathetic reactivity during the speech delivery task that was faster than that of the HPA axis in the presence of threatening stimuli.

Assessment of the hypothalamic-pituitary adrenal axis (HPA) Collection of salivary cortisol samples was performed using Salivette ${ }^{\circledR}$ Cortisol (Sarstedt, Numbrecht, Germany, Ref.51.1534), which consists of two small tubes, one of them containing a small piece of cotton. Participants chewed the cotton for about $60 \mathrm{~s}$, after which it was introduced into the salivette for analysis. Samples were analyzed at the San Cecilio University Hospital, using the electrochemiluminescence immunoassay "ECLIA" method. This method is designed for use in Roche Elecsys 1010/2010 automated analyzers and in the Elecsys MODULAR ANALYTICS E170 module. Salivary cortisol samples were obtained at four collection times in the study: preexposure cortisol, post-exposure cortisol, post-exposure cortisol +10 min and post-exposure cortisol +20 min.

\section{Statistical analyses}

Initially, Student's $t$-tests were used to check for differences between groups (goggles vs. screen) in terms of sociodemographic (age and education level), and psychologic (SV, PSS, and SCL-90-R) variables. Chi-square analyses were performed to check for differences between groups in sex, use of tobacco, and contraception.

Next, we conducted a $2 \times 2 \times 2$ ANCOVA in order to test for differences in conductance between the two types of VR presentation protocols (goggles vs. screen), the sexes (males vs. females), and the time of data acquisition (anticipatory stress vs. speech delivery). Baseline levels of conductance for each participant were used as a covariate.

Additionally, we carried out a $2 \times 2 \times 3$ ANCOVA to analyze if there were significant differences in cortisol levels associated with the two types of VR protocols (goggles vs. screen), the sexes (males vs. females), and the time of cortisol sampling (post-exposure, post-exposure $+10 \mathrm{~min}$ and postexposure $+20 \mathrm{~min}$ ). Pre-exposure levels of cortisol for each participant were used as a covariate.

The Greenhouse-Geisser correction was applied to all analyses involving repeated measures and sphericity violations. Also, in the case of cortisol responses, Bonferroni post-hoc analyses were conducted when analyzing within-group differences across time during the experimental session.

When significant Group $\times$ Sampling Time interactions were found, follow-up Student's $t$-tests were carried out to determine whether there were differences in either skin conductance or cortisol level between groups at each sampling time. 


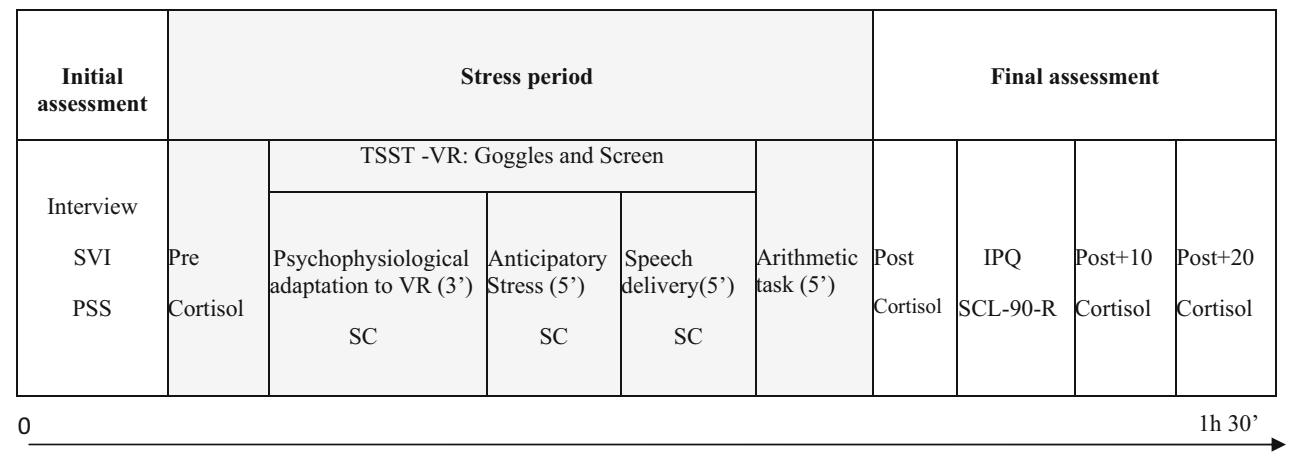

Fig. 2 Diagrammatic representation of the protocol of a virtual reality adaptation of the Trier Social Stress Test (TSST-VR). Note: SVI: Stress Vulnerability Inventory; PSS: Perceived Stress Scale; TSST -VR: Trier Social Stress Test adapted to Virtual Reality; Pre-cortisol: pre-exposure cortisol; Post-cortisol: post-exposure cortisol; Post +10 cortisol: cortisol at $10 \mathrm{~min}$ after exposure; Post +20 cortisol: cortisol at $20 \mathrm{~min}$ after exposure; SC: skin conductance; IPQ: Igroup Presence Questionnaire; SCL90-R: Symptom Checklist SCL-90-R
Finally, we conducted a $2 \times 2$ ANOVA that included the type of VR protocol (goggles vs. screen) and sex (males vs. females) to analyze the four subscales of the IPQ: global, spatial presence, involvement, and experienced realism. Significant interactions were further examined by means of Student's $t$-tests. Further, correlation analyses were used to test the relationship between the stress variables (PSS and SVI) and the IPQ.

\section{Results}

\section{Sample description}

Sociodemographic and psychologic data for the participants can be found in Table 1. Results showed significant statistical differences between type of protocol in the PSS, with the screen group scoring higher than the goggles

Table 1 Means (M) and standard deviations (SD) of sociodemographic and psychologic variables for the participants in both groups

\begin{tabular}{|c|c|c|c|c|}
\hline \multirow[t]{2}{*}{ Variables } & \multicolumn{2}{|l|}{ Groups } & \multicolumn{2}{|c|}{ Analyses } \\
\hline & Goggles $\mathrm{M} \pm \mathrm{SD}$ & Screen $\mathrm{M} \pm \mathrm{SD}$ & $t / \chi^{2}$ & $p$ \\
\hline Age (years) & $21.9 \pm 5$ & $20.4 \pm 1.25$ & 1.36 & 0.181 \\
\hline Education Level (years) & $14 \pm 0.00$ & $14 \pm 0.00$ & -1.07 & 0.290 \\
\hline \multicolumn{5}{|l|}{$\operatorname{Sex}\left(\%, \chi^{2}\right)$} \\
\hline Males & $45.83 \%$ & $25 \%$ & 1.4 & 0.230 \\
\hline Females & $54.17 \%$ & $75 \%$ & & \\
\hline \multicolumn{5}{|l|}{ Smoking $\left(\%, \chi^{2}\right)$} \\
\hline Males & $27.27 \%$ & $0 \%$ & 2.7 & 0.100 \\
\hline Females & $30.77 \%$ & $6.5 \%$ & & \\
\hline Contraceptives $\left(\%, \chi^{2}\right)$ & $4.33 \%$ & $1.3 \%$ & 2.6 & 0.140 \\
\hline Perceived Stress Scale & $20.08 \pm 10.03$ & $26.90 \pm 5.15$ & -2.81 & $0.007 *$ \\
\hline Stress Vulnerability Inventory & $4.91 \pm 3.92$ & $6.62 \pm 4.07$ & -1.43 & 0.161 \\
\hline \multicolumn{5}{|l|}{ Symptom Checklist SCL-90-R } \\
\hline Somatization & $54.20 \pm 11.39$ & $55.52 \pm 6.83$ & -0.46 & 0.647 \\
\hline Obsessions and compulsions & $58.96 \pm 9.32$ & $61.71 \pm 6.02$ & -1.16 & 0.253 \\
\hline Interpersonal sensitivity & $57.96 \pm 12.99$ & $59.81 \pm 7.51$ & -0.57 & 0.569 \\
\hline Depression & $48.56 \pm 14.30$ & $54.09 \pm 6.03$ & -1.65 & 0.107 \\
\hline Anxiety & $52.87 \pm 10.21$ & $54.38 \pm 7.89$ & -0.55 & 0.587 \\
\hline Hostility & $50.37 \pm 11.40$ & $52.05 \pm 8.13$ & -0.56 & 0.579 \\
\hline Phobic anxiety & $43.71 \pm 13.12$ & $42.19 \pm 9.23$ & 0.44 & 0.660 \\
\hline Paranoia & $57.50 \pm 10.09$ & $55.38 \pm 8.76$ & 0.75 & 0.459 \\
\hline Psychoticism & $52.62 \pm 14.11$ & $53.95 \pm 8.50$ & -0.37 & 0.709 \\
\hline
\end{tabular}

Note: $\chi^{2}:$ Chi-square analysis; $t:$ Student's $t$-test value; ${ }^{*} \mathrm{p} \leq 0.05$ 
group. Thus, PSS scores were added as covariate in the following analyses.

Sympathetic reactivity: Skin conductance

Results showed a main effect of type of VR protocol $[\mathrm{F}(1,42)$ $=4.440 ; \mathrm{p}=0.041]$, with the goggles condition evoking higher sympathetic activity overall.

A significant interaction between type of VR protocol and assessment time $[F(1,42)=3.959 ; \mathrm{p}=0.050]$ revealed a higher activation between anticipatory stress $(\mathrm{M}=2.85 ; \mathrm{SD}=1.95)$ and speech delivery $(\mathrm{M}=3.81 ; \mathrm{SD}=2.72)$ in the goggles group than in the screen group (anticipatory stress: $\mathrm{M}=2.98 ; \mathrm{SD}=$ 1.20; speech delivery: $\mathrm{M}=3.34 ; \mathrm{SD}=1.10$ ) Fig. 3 .

Activation of the hypothalamic-pituitary-adrenal axis (HPA): Salivary cortisol

In terms of HPA axis activation, there was no evidence of any main effect or an interaction between the level of activation of the HPA axis and the type of TSST-VR protocols, goggles or screen projection, at any of the cortisol extraction times.

Finally, results showed that there was no interaction between sex and cortisol secretion levels, regardless of whether the TSST-VR was presented by means of goggles or projected on a screen.

Immersion in the virtual environment (IPQ)

Statistical analyses demonstrated statistically significant differences in the involvement subscale of the IPQ as a function of the sex of the participant (independent of VR protocol used), with males ( $\mathrm{M}=-1.06 ; \mathrm{SD}=5.43)$ endorsing lower levels of involvement than females $(\mathrm{M}=2.92 ; \mathrm{SD}=3.50)$ $[\mathrm{F}(1,42)=7.29 ; \mathrm{p}=0.01]$.

On the experienced realism subscale, there was a significant interaction between sex and type of TSST-VR protocol $[\mathrm{F}(1,42)=6.63 ; p=0.014]$, such that males obtained higher

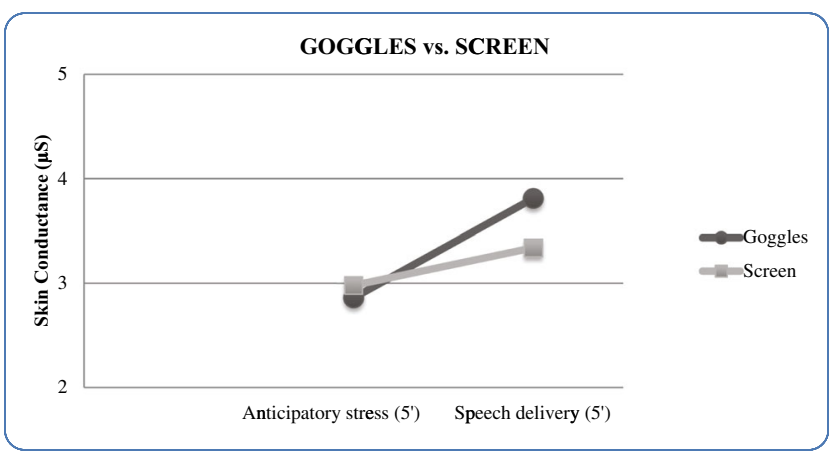

Fig. 3 Skin conductance for two protocols of (TSST-VR) delivery (Goggles vs. Screen), recorded at two points in the experiment scores than females only on the screen projection condition (males: $\mathrm{M}=-1.66$; $\mathrm{SD}=2.16$ vs. females: $\mathrm{M}=-4.26$; $\mathrm{SD}=2.05)$. No significant differences were found on the global and spatial presence subscales.

Significant correlations were found between scores on the PSS and on the IPQ subscales involvement $(r=0.348 ; \quad p$ $=0.02)$ and spatial presence $(r=0.519 ; p<0.001)$. Similarly, SVI scores correlated with those obtained on the IPQ subscales involvement $(r=0.298 ; p=0.049)$ and spatial presence $(r=0.428 ; p=0.004)$.

\section{Discussion}

The objective of this study was twofold: firstly, we aimed to determine which of two protocols of the TSST-VR, whether a 3D virtual audience projected onto goggles or a large screen, produced greater sympathetic reactivity and activation of the HPA axis. Secondly, we sought to find out whether males and females showed distinct levels of activation of the sympathetic nervous system and HPA axis in these two TSST-VR protocols.

In terms of activation of the HPA axis, we found no statistical differences between the two TSST-VR protocols: goggles versus screen. However, sympathetic reactivity, as measured by skin conductance, did vary across presentation protocols, being higher in the goggles projection condition than in the screen condition. This effect could not be attributed to the level of immersion associated with each of the experimental conditions.

Here, it is worth emphasizing that, although an adequate level of immersion in the virtual environment seems indispensable to activate the stress response axes, some studies (e.g., Santos-Ruiz et al., 2010) have failed to find a significant relationship between the HPA axis and the level of immersion in the virtual environment. In the present study, we did not find a significant relationship of the HPA axis and sympathetic reactivity with the level of immersion in the virtual environment. In fact, although females obtained higher scores in the involvement subscale of the IPQ, regardless of VR protocol, there were no significant sex differences associated with HPA axis activation and sympathetic reactivity. Similar results were found with the screen protocol in male participants that, despite obtaining higher scores on the experienced realism subscale of the IPQ, did not show higher sympathetic activation either. It seems that the goggles elicited significantly higher conductance values overall, but this effect was not associated with any subscale of the IPQ and did not vary across sexes.

On the other hand, these results are in agreement with the recent study by Ling, Brinkman, Nefs, Qu, and Heyndericky (2013) in which real-size screen presentations of a virtual classroom were associated with a greater sense of presence when compared to real- or reduced-size TV projections.

A positive correlation was found between stress levels (as measured by the PSS and VSI) and the IPQ involvement and 
spatial presence subscales, independent of VR protocol used (goggles or screen) or the participant's sex. In other words, those participants reporting higher stress levels were also more likely to feel involved and spatially present during the task, regardless of the manner in which the virtual audience was presented. These results concur with those of Ling, Nefs, Morina, Heynderickx, and Brinkman (2014), who found a correlation between sense of presence and anxiety, but not for sex or type of projection of the virtual environment (e.g., field of view, degree of freedom of the tracker, etc.). Since we did not measure anxiety levels in the present study, we cannot say whether a greater spatial presence leads to a higher level of anxiety, but we did find that those participants with higher levels of stress prior to the TSST-VR protocol showed a higher level of involvement and a greater sense of spatial presence, as measured by the IPQ.

The results showed sex differences in that females manifested a greater degree of involvement than males, regardless of the VR protocol used. In contrast, females exposed to the 3D audience of the TSST-VR by means of the screen projection manifested less experienced realism than males. Such sex differences need to be considered in designing research that uses VR methodologies, given that differences between males and females could be erroneously attributed to distinct HPA axis responses when, in fact, they are due to differences in the degree of VR immersion of each gender. In fact, the two aims of this study stemmed from results such as those reported by Kotlyar et al. (2008), showing that the TSST-VR produces sympathetic activation and engages the HPA axis. Similarly, Kelly et al. (2007) concluded that the TSST-VR activates the stress response and constitutes a useful tool in that it solves some of the limitations associated with the traditional TSST.

In recent years, there have been reports of various investigations demonstrating the utility of the TSST-VR. Nevertheless, despite common agreement on the suitability of the VR version of this task in engaging the axes mediating the stress response, certain controversial issues still remain. One of these issues pertains to the presentation of the virtual environments that are common to most. Some investigators have projected 3D environments onto large screens (Santos-Ruiz et al., 2010), while others have used goggles (Jönsson et al., 2009; Kelly et al., 2007). Despite these differences, no study has contrasted the various versions of the TSST-VR to detect which form of presentation results in a greater response to the stress generated by the task. Additionally, although it is well known that the traditional TSST evokes more activation in males than in females (Foley \& Kirschbaum, 2010; Hemmeter et al., 2005; Williams et al., 2004), sex differences in response to the TSST-VR have not been systematically studied. The results obtained in this study provide some answers to these questions.

It should be noted, however, that use of the VR goggles could have caused cybersickness, according to theories like the Sensory Conflict Theory, the Poison Theory, and the
Postural Instability Theory (La Viola Jr., 2000). Still, no member of the goggle presentation group reported symptoms of cybersickness, nor were there any indications of cybersickness in the skin conductance records.

The present study has limitations that need to be taken into account when considering the study and its findings. Firstly, given that one of our objectives was to characterize sex differences in the two versions of the TSST-VR used here, it would have been desirable to have an equal number of males and females. Likewise, it would have been preferable to count on matched levels of perceived stress across groups at the baseline level, since the initial differences could be selectively influencing stress responses at both the sympathetic and HPA axis levels.

In summary, our results appear to support the use of the TSST-VR, whether it is delivered via goggles or via a large screen projection, as a useful paradigm to investigate the modulation of the principal axes that intervene in the stress response. More concretely, if the aim of the study is to specifically investigate one of the two axes, it seems that it would be preferable to use a goggles protocol to activate the sympathetic nervous system and projection of the $3 \mathrm{D}$ image onto a screen to activate the HPA axis, particularly if participants are mainly females. In investigating the stress response in both axes and mostly with males, our results seem to favor the use of the goggles protocol. Finally, if the study were to include both stress response axes and both sexes, our findings would indicate that the use of a 3D image projected onto a large screen would be the recommended TSST-VR protocol.

Acknowledgments This study is a part of a Thesis Doctoral and was supported by the I+D Project "PSI2010-15780" of the Spanish Ministry of Science and Innovation. The Ministry was not involved in study design, in the collection, analysis and interpretation of data, in the writing of the report, nor in the decision to submit the paper for publication.

\section{References}

Al'Absi, M., Bongard, S., Buchanan, T., Pincomb, G. A., Licinio, J., \& Lovallo, W. R. (1997). Cardiovascular and neuroendocrine adjustment to public speaking and mental arithmetic stressors. Psychophysiology, 34, 266-275.

Annerstedt, M., Jönsson, P., Wallergård, M., Johansson, G., Karlson, B., Grahn, P., \& Währborg, P. (2013). Inducing physiological stress recovery with sounds of nature in a virtual reality forest-results from a pilot study. Physiology \& Behavior, 118, 240-250. doi: 10.1016/j. physbeh.2013.05.023

Beech, H. R., Burns, L. E., \& Scheefield, B. F. (1986). Tratamiento del estrés. Un enfoque comportamental. Madrid: Ed. Alambra.

Brenner, K., Liu, A., Laplante, D.P., Lupien, S., Pruessner, J.C., Ciampi, A., \& King, S. (2009). Cortisol response to a psychosocial stressor in schizophrenia: blunted, delayed, or normal? . Psychoneuroendocrinology, 34(6), 859-868. doi:10.1016/j.psyneuen. 2009.01.002

Buske-Kirschbaum, A., Jobst, S., Wustmans, A., Kirschbaum, C., Rauh, W., \& Hellhammer, D. (1997). Attenuated free cortisol response to 
psychosocial stress in children with atopic dermatitis. Psychosomatic Medicine, 59(4), 419-426.

Chrousos, G. P. (2000). The role of stress and the hypothalamic-pituitary-adrenal axis in the pathogenesis of the metabolic syndrome: Neuro-endocrine and target tissue-related causes. International Journal of Obesity, 24(Suppl. 2), S50-S55.

Cohen, S., Kamarak, T., \& Mermeistein, R. (1983). A global measure of perceived stress. Journal of Health and Social Behavior, 24, 385396.

De las Cuevas, C., González de Rivera, J. L., Henry- Benitez, M., Monterrey, A. L., Rodríguez-Pulido, F., \& Gracia- Marco, R. (1991). Análisis factorial de la versión española del SCL-90-R en la población general. Anales de Psiquiatría, 7, 93-96.

Derogatis, L. R. (1994). Symptom checklist 90. Administration Scoring and Procedures Manual. Minneapolis: National Computer Systems Inc.

Esler, M., Eikelis, N., Schlaich, M., Lambert, G., Alvarenga, M., Kaye, D., \& Lambert, E. (2008). Human sympathetic nerve biology: parallel influences of stress and epigenetics in essential hypertension and panic disorder. Annals of the New York Academy of Sciences, 1148, 338-348. doi:10.1196/annals.1410.064

Fiocco, A. J., Joober, R., \& Lupien, S. J. (2007). Education modulates cortisol reactivity to the Trier Social Stress Test in middle-aged adults. Psychoneuroendocrinology, 32, 1158-1163.

Foley, P., \& Kirschbaum, C. (2010). Human hypothalamus-pituitaryadrenal axis responses to acute psychosocial stress in laboratory settings. Neuroscience and Biobehavioral Reviews, 35, 91-96. doi: 10.1016/j.neubiorev.2010.01.010

González de Rivera, J. L., \& De las Cuevas, C. (1988). Versión española del cuestionario SCL-90-R. Tenerife: Universidad de la Laguna (policopiado).

Hemmeter, U., Störmer, R., Mager, R., Kuntze, M., Mueller-Spahn, F., Hennig, J., \& Bullinger, A. (2005). Modification of virtual reality combined with a mental task stimulates cortisol in volunteers. Neuropsychobiology, 51, 165-172. doi:10.1159/ 000085208

Jönsson, P., Wallergård, M., Österberg, K., Hansen, Å. M., Johansson, G., \& Karlson, B. (2010). Cardiovascular and cortisol reactivity and habituation to a virtual reality version of the Trier Social Stress Test: A pilot study. Psychoneuroendocrinology, 35, 1397-1403. doi:10.1016/j.psyneuen.2010.04.003

Kelly, O., Matheson, K., Martinez, A., Merali, Z., \& Anisma, H. (2007). Psychosocial stress evoked by a virtual audience: Relation to neuroendocrine activity. Cyberpsychology \& Behavior, 5, 655-662.

Kirschbaum, C., Bartussek, D., \& Strasburger, C. J. (1992). Cortisol responses to psychological stress and correlations with personality traits. Personality and Individual Differences, 13, 1353-1357.

Kirschbaum, C., Pirke, K. M., \& Hellhammer, D. H. (1993). The "Trier Social Stress Test" - A tool for investigating psychobiological stress responses in a laboratory setting. Neuropsychobiology, 28, 76-81

Kotlyar, M., Donahue, C., Thuras, P., Kushner, M. G., O'Gorman, N., Smith, E. A., \& Adson, D. E. (2008). Physiological response to a speech stressor presented in a virtual reality environment. Psychophysiology, 45, 1034-1037. doi:10.1111/j.1469-8986.2008. 00690

Kudielka, B. M., Hellhammer, J., Hellhammer, D. H., Wolf, O. T., Pirke, K. M., Varadi, E., \& Kirschbaum, C. (1998). Sex differences in endocrine and psychological responses to psychosocial stress in healthy elderly subjects and the impact of a 2-week dehydroepiandrosterone treatment. Journal of Clinical Endocrinology and Metabolism, 83, 1756-1761.

Kudielka, B. M., \& Kirschbaum, C. (2005). Sex differences in HPA axis responses to stress: A review. Biological Psychology, 69, $113-132$.
La Viola, J. J., Jr. (2000). A discussion of cybersickness in virtual environments. SIGCHI Bulletin, 32(1), 47-56.

Ling, Y., Brinkman, W. P., Nefs, H. T., Qu, C., \& Heyndericky, I. (2013). The effect of perspective on presence and space perception. PLoS ONE, 8(11), e78513. doi:10.1371/journal.pone. 0078513

Ling, Y., Brinkman, W. P., Nefs, H. T., Qu, C., \& Heyndericky, I. (2012). Effects of stereoscopic viewing on presence, anxiety, and cybersickness in a virtual reality environment for public speaking. Presence, 21(3), 254-267. doi:10.1162/PRES_a 00111

Ling, Y., Nefs, H. T., Morina, N., Heynderickx, I., \& Brinkman, W. P. (2014). A meta-analysis on the relationship between self-reported presence and anxiety in virtual reality exposure therapy for anxiety disorders. PLoS ONE, 9(5), e96144. doi:10.1371/journal.pone. 0096144

Malbos, E., Boyer, L., \& Lançon, C. (2013). Virtual reality in the treatment of mental disorders. Presse Médicale, 42(11), 1442-1452. doi: 10.1016/j.lpm.2013.01.065

Morina, N., Brinkman, W., Hartanto, D., \& Emmelkamp, P. M. G. (2014). Sense of presence and anxiety during virtual social interactions between a human and virtual humans. PeerJ, 2, e337.

Nicolson, N., Storms, C., Ponds, R., \& Sulon, J. (1997). Salivary cortisol levels and stress reactivity in human aging. The Journals of Gerontology Series A: Biological Sciences and Medical Sciences, 52(2), M68-M75.

Parker, K. J., Schatzberg, A. F., \& Lyons, D. M. (2003). Neuroendocrine aspects of hypercortisolism in major depression. Hormones and Behavior, 43, 60-66.

Pawlak, C. R., Jacobs, R., Mikeska, E., Ochsmann, S., Lombardi, M. S., Kavelaars, A., \& Schedlowski, M. (1999). Patients with systemic lupus erythematosus differ from healthy controls in their immunological response to acute psychological stress. Brain, Behavior, and Immunity, 13(4), 287-302.

Pertaub, D. P., Slater, M., \& Barker, C. (2002). An experiment on public speaking anxiety in response to three different types of virtual audience. Presence: Teleoperators and Virtual Environments, 11(1), 6878. doi:10.1162/105474602317343668

Pruessner, J. C., Gaab, J., Hellhammer, D. H., Lintz, D., Schommer, N., \& Kirschbaum, C. (1997). Increasing correlations between personality traits and cortisol stress responses obtained by data aggregation. Psychoneuroendocrinology, 22(8), 615-625.

Remor, E. (2006). Psychometric properties of a European Spanish version of the Perceived Stress Scale (PSS). The Spanish Journal of Psychology, 29, 86-93.

Remor, E., \& Carrobles, A. (2001). Versión española de la escala de estrés percibido (PPS-14): Estudio psicométrico en una muestra VIH+. Ansiedad y Estrés, 7(2), 195-201.

Robles-Ortega, H., Peralta-Ramírez, M. I., \& Navarrete-Navarrete, N. (2006). Validación de la versión española del Inventario de Vulnerabilidad al Estrés de Beech, Burns y Scheffield. Avances en Psicología de la Salud. Granada: Ediciones Sider.

Santos-Ruiz, A., Peralta-Ramírez, M. I., García- Ríos, M. C., Muñoz, M. A., Navarrete-Navarrete, N., \& Blázquez-Ortiz, A. (2010). Adaptation of the trier social stress test to virtual reality: Psychophysiological and neuroendocrine modulation. Journal of Cybertherapy \& Rehabilitation, 3, 405-415.

Sjörs, A., Larsson, B., Karlson, B., Osterberg, K., Dahlman, J., \& Gerdle, B. (2010). Salivary cortisol response to acute stress and its relation to psychological factors in women with chronic trapezius myalgia-a pilot study. Psychoneuroendocrinology, 35(5), 674-685. doi:10.1016/j. psyneuen.2009.10.007

Schubert, T., Friedmann, F., \& Regenbrecht, H. (2001). The experience of presence: Factor analytic insights. Presence: Teleoperators and Virtual Environments, 10, 266-281. 
Shirotsuki, K., Izawa, S., Sugaya, N., Yamada, K.C., Ogawa, N., Ouchi, Y., \& Nomura, S. (2009). Salivary cortisol and DHEA reactivity to psychosocial stress in socially anxious males. The International Journal of Psychophysiology, 72, 198-203.

Vinkers, C.H., Penning, R., Hellhammer, J., Verster, J.C., Klaessens, J.H., Olivier, B.M., \& Kalkman, C.J. (2013).
The effect of stress on core and peripheral body temperature in humans. Stress, 5, 520-530. doi:10.3109/10253890.2013. 807243

Williams, R. A., Hagerly, B. M., \& Brooks, G. (2004). Trier Social Stress Test. A method for use in nursing research. Nursing Research, 53, 277280. 